\title{
New pumping scheme for high gain and low noise figure in an erbium-doped fiber amplifier
}

\author{
V. Sinivasagam, ${ }^{1,3 a)}$ Mustafa A. G. Abushagur, ${ }^{1,2}$ K. Dimyati, ${ }^{3}$ \\ and F. Tumiran ${ }^{1}$ \\ ${ }^{1}$ Photronix (M) Sdn. Bhd., G05, 2300 Century Square, Jalan Usahawan, 63000 \\ Cyberjaya, Selangor, Malaysia \\ ${ }^{2}$ Microsystems Engineering, Rochester Institute of Technology, Rochester, NY 14623, \\ USA \\ ${ }^{3}$ Department of Electrical Engineering, University of Malaya, 50603 Kuala Lumpur \\ Malaysia
}

a)vijay_photonics@hotmail.com

\begin{abstract}
We propose a new pumping method for optical fiber amplifiers at $1480 \mathrm{~nm}$ and $980 \mathrm{~nm}$ that is able to provide high gain and low noise-figure. The gain and noise figure obtained were $38.3 \mathrm{~dB}$ and $5.5 \mathrm{~dB}$ with an improvement of $3.8 \mathrm{~dB}$ and $1.4 \mathrm{~dB}$, respectively, at $1550 \mathrm{~nm}$, for $-30 \mathrm{dBm}$ input power when compared to its counterpumped design. This design also delivers higher gain and lower noise figure when compared to the common bi-directional dual-pumped designs. A power conversion efficiency of $44.5 \%$ was achieved in this design.
\end{abstract}

Keywords: Optical amplifiers, pumping methods, erbium-doped

Classification: Photonics devices, circuits, and systems

\section{References}

[1] C. M. Weinert, R. Ludwig, W. Pieper, H. G. Weber, D. Breuer, K. Petermann, and F. Kuppers, " $40 \mathrm{~Gb} / \mathrm{s}$ and $4 \times 40 \mathrm{~Gb} / \mathrm{s}$ TDM/WDM Standard Fiber Transmission," J. Lightwave Technol., vol. 17, no. 11, pp. 2276-2284, Nov. 1999.

[2] B. Pedersen, M. L. Dakss, B. A. Thompson, W. J. Miniscalco, T. Wei, and L. J. Andrews, "Experimental and theoretical analysis of efficient erbiumdoped fiber power amplifiers," IEEE Photon. Technol. Lett., vol. 3, no. 12, pp. 1085-1087, Dec. 1991.

[3] Uh-Chan Ryu, K. Oh Woojin, and U. C. Paek, "Inherent Enhancement of Gain Flatness and Achievement of Broad Gain Bandwidth in ErbiumDoped Silica Fiber Amplifier," IEEE J. Quantum Electron., vol. 38, no. 2, pp. 149-161, Feb. 2002.

[4] B. V. Caenegem, "Optical Networks: Status and Trends," Proc. TERENA-NORDUnet Networking Conference, Sweden, June 1999.

[5] P. C. Becker, N. A. Olsson, and J. R. Simpson, Erbium-Doped Fiber Amplifiers: Fundamentals and Technology, Academic Press, p. 354, San Diego, 
1999.

[6] E. Desurvire, Erbium Doped Fiber Amplifiers: Principle and Applications, John Wiley \& Sons, New York, 1994.

[7] S. Milo, R. F. Souza, M. B. C. Silva, E. Conforti, and A. C. Bordonalli, "An EDFA theoretical analysis considering different configurations and pumping wavelengths," Proc. IMOC, vol. 1, pp. 105-110, Sept. 2003.

[8] M. E. Bray, T. J. Reid, K. P. Jones, and M. P. Poettcker, "Model to predict spectral shape of an hybrid $980 \mathrm{~nm}-1480 \mathrm{~nm}$ pumped erbium doped fibre amplifier," Proc. OFC/IOOC '99, vol. 2 , pp. 111-113, Feb. 1999.

\section{Introduction}

Broadband applications have risen; however, demands from end-users will soon outgrow the capacity of the present optical communication networks. Therefore, high bandwidth based devices will play a major role in the next generation networks. Erbium-doped fiber amplifier (EDFA) has been extremely compatible to both low and high bandwidth communication networks [1]. In order to cope with the bandwidth demand, new EDFAs has been proposed to provide high gain and to fully utilize the redundant fiber bandwidth $[2,3]$. Wavelength division multiplexing (WDM) systems with terabits per second capability together with EDFA has been installed in submarine networks [4] anticipating this bandwidth explosion. Limitations such as amplified spontaneous noise (ASE) peaking in long-haul optical communication networks that leads to gain and noise figure degradation in an EDFA has to be tackled [5]. Thus, the need for EDFAs with high gain and low noise has been of interest to all WDM system manufacturers.

Pumping scheme in an EDFA is an important factor in determining its type (booster, in-line and pre-amplifier) and performance (gain and noisefigure). In a co-pumped design, low gain and low noise figure is achieved in contrast to the counter-pumped design where the gain and noise figure is relatively higher [6]. As a compromise bi-directional pumping scheme were introduced to achieve higher gain with notably high noise figures $=[7]$.

We propose a new pumping scheme to achieve high gain with low noise figure by using a dual co-pumping (DP) scheme utilizing both $980 \mathrm{~nm}$ pump and $1480 \mathrm{~nm}$ pump in an EDFA. Two related designs under co- and counterpropagating signal conditions were investigated and compared with standard bi-directional pumping (BP) scheme designs [8]. Results obtained show that the DP scheme with co-propagating signal produced the highest gain and lowest noise when compared with the other designs mentioned.

\section{Design and Experimental Procedure}

The schematics of four different designs of the EDFA are shown Fig. 1 (a), (b), (c) and (d) respectively. Fig. 1 (a) and (b) shows a standard BP scheme based EDFA designs under co- and counter-propagating signal conditions termed as co-BP and counter-BP respectively. The co- and counter- terminology 
used here for the $\mathrm{BP}$, is based on signal propagation direction relative to the $980 \mathrm{~nm}$ pump. The terminologies selected were merely to differentiate the designs and not reference to any terminology standards. While the proposed new pumping scheme utilizing DP scheme is shown in Fig. 1(c) and (d) at co- and counter- propagating signal conditions which is termed as co-DP and counter-DP respectively.

In order to perform a comparison, all the design setups comprise similar components, erbium-doped $\left(\mathrm{Er}^{3+}\right)$ fiber (EDF) type and length and machine splice losses. The pump sources were $980 \mathrm{~nm}$ and $1480 \mathrm{~nm}$ laser diodes fixed at a power of $110 \mathrm{~mW}$ and $120 \mathrm{~mW}$ respectively. The EDF length was $25 \mathrm{~m}$ with a nominal $\mathrm{Er}^{3+}$ concentration of $240 \mathrm{ppm}$ and the absorption at 1530 was $5.6 \mathrm{~dB} / \mathrm{m}$. However, the measured total pump input loss for the BP and the DP schemes were different even though the signal input loss was similar at $0.1 \mathrm{~dB}$. The latter scheme has a larger pump input loss mainly contributed by the high splice loss experience by the $1480 \mathrm{~nm}$ pump between the $1480 / 1550 \mathrm{~nm}$ wavelength selective coupler (WSC) and the $980 / 1550 \mathrm{~nm}$ WSC due to difference in fiber type (SMF-28 x Flexcor 1060) and fiber cutoff wavelength. The pump input loss was $1.2 \mathrm{~dB}$ and $1.8 \mathrm{~dB}$ for the $\mathrm{BP}$ and DP scheme respectively.

All the designs operate in the C-band region and was characterized from $1530 \mathrm{~nm}$ to $1570 \mathrm{~nm}$ with $2 \mathrm{~nm}$ step with the input signal source from a tunable C- to L-band laser source and measurement of gain, noise figure and output power was done using an optical amplifier analyzer (ANDO, Model: AQ8423Z). Accurate noise figure values were obtained via the pulse mode technique used by the analyzer. The input signal was fixed at $-30 \mathrm{~d}$ Bm to compare the small signal gain of the EDFA designs.

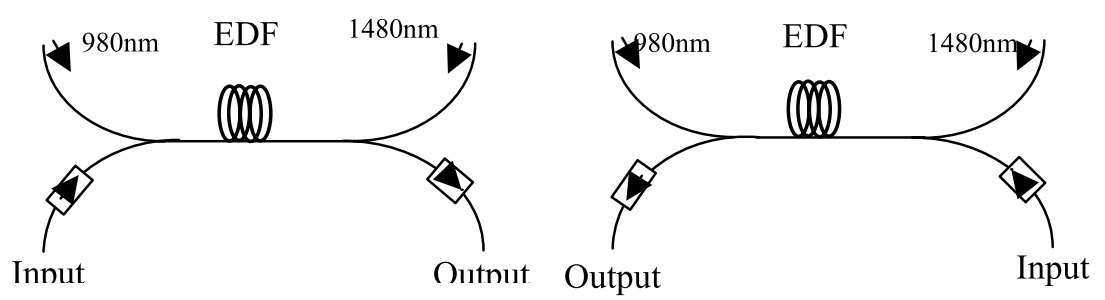

(a) Bi-directional pumping co-signal (co-BP)

(b) Bi-directional pumping counter-signal (counter-BP)

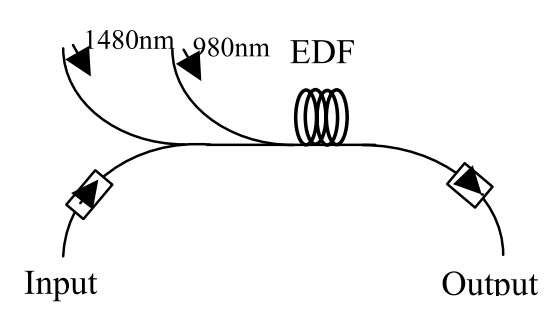

(c) Co-dual pumping co-signal (co-DP)

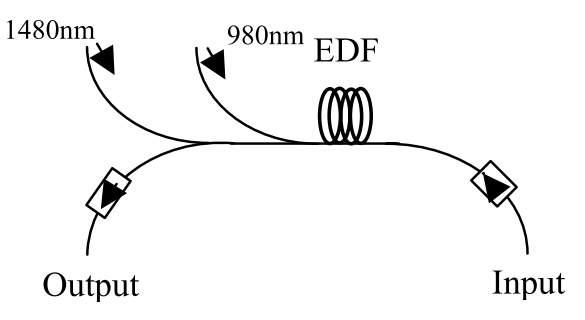

(d) Co-dual pumping counter-signal (counter-DP)

Fig. 1. Schematics of the EDFA designs with different pumping schemes 


\section{Results and Discussion}

Gain and noise-figure characteristics for all the four designs as a function of wavelength are shown in Fig. 2. The co- and counter-BP designs have almost similar gain values over the entire wavelength range. This indicates that in the BP scheme the EDF is fully pumped and have minimal effect to the direction of signal propagation to its gain performance. Whereas, for the DP scheme, gain variation between the two designs is obvious with the co-DP having higher gain as compared to the counter-DP and the former having the highest gain among the four designs. A maximum gain of $38.3 \mathrm{~dB}$ was achieved from the co-DP design with a gain difference of $3.8 \mathrm{~dB}$ when compared to the counter-DP design. These results opposed to a standard EDFA characteristics where the gain and noise figure are higher for the counter-pumped design as compared to the co-pumped design. This noticeable effect in the DP-scheme is due to the pumping scheme practiced here where, counter-propagating signals experience loss at the unbleached region due to absorption at the end of the EDF which lacks pump power. For the co-propagating signals strong amplification at the beginning of the EDF overwhelms this signal absorption at the end of the fiber thus providing higher gain than the counter propagating signal. Even though the gain difference between the co-DP and the $\mathrm{BP}$ designs is small but we believe by reducing the splice loss between the input WDMs of the co-DP, the gain can be further improved. The combination of $1480 \mathrm{~nm}$ and $980 \mathrm{~nm}$ for the dual pumping scheme clearly contributes to the gain and output power besides efficiency of the co-DP design.

The co-DP has the lowest noise figure values for almost the entire operational region of the C-band $(1535 \mathrm{~nm}-1565 \mathrm{~nm})$ comparatively. As seen from Fig. 1, only the co-DP design has its signal and pumps propagating in

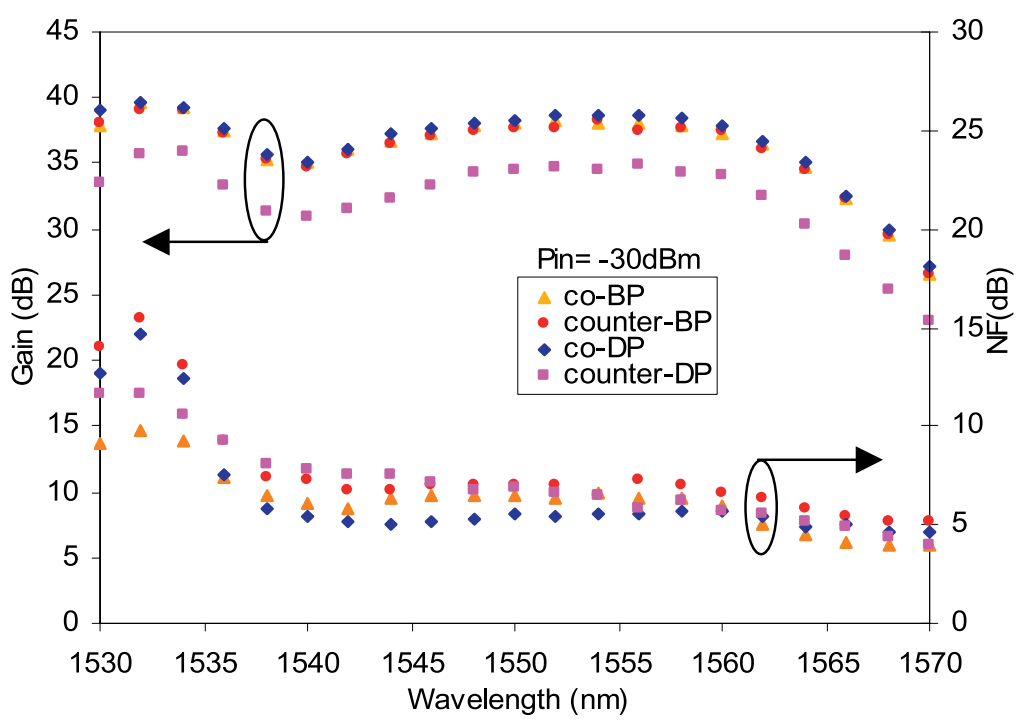

Fig. 2. Gain and noise figure performance for four different designs 
the same direction whereas for the co-BP, as mentioned earlier, the $1480 \mathrm{~nm}$ pump propagates opposite to the signal direction and similarly the signal and pump direction are opposite for the other counter designs. This copumping technique has proven to deliver lower noise figure in single pump EDFA and has similar advantages on this dual co-pumped design as well. Noise figure measured at $1550 \mathrm{~nm}$ was $5.5 \mathrm{~dB}, 6.9 \mathrm{~dB}, 6.4 \mathrm{~dB}$ and $7.0 \mathrm{~dB}$ for the co-DP, counter-DP, co-BP and counter-BP designs respectively. With this new pumping scheme in a co-DP, a noise figure improvement of $1.4 \mathrm{~dB}$ and $0.9 \mathrm{~dB}$ is achieved as compared to counter-DP and the co-BP designs. In the co-DP design, strong signal amplification at the beginning of the EDF contributes to strong pump to signal conversion and thus reducing undesired ASE from depleting the population inversion of the EDF, providing low noise at the output of this design.

The high gain and low noise characteristics of the co-DP design introduced in this paper has its advantages to be used as an excellent pre-amplifier in DWDM long-haul networks. Besides, at high input signal of $-8 \mathrm{dBm}$ the co-DP could easily produce an output power of $19.2 \mathrm{dBm}$ with its power conversion efficiency (PCE) at $44.5 \%$ which also has its potential to be a good choice of booster applications in communication systems.

\section{Conclusion}

A dual co-pumped pumping scheme in a C-band EDFA has been proposed and investigated. The proposed co-DP design delivers higher gain and lower noise figure when compared to the standard bi-directional designs or the counter-DP design. Gain and noise figure improvement of $3.8 \mathrm{~dB}$ and $1.4 \mathrm{~dB}$ was obtained comparatively. The new pumping scheme in the co-DP design also produced high output power of $19.2 \mathrm{dBm}$ and a PCE of $44.5 \%$ at $-8 \mathrm{dBm}$ signal input power. The design shows great potential as a pre-amplifier at low input levels and as well as booster at high input levels, an ideal solution for DWDM networks.

\section{Acknowledgments}

The authors would like to thank the Multimedia Development Corporation (MDC) for their financial support of this research under the MGS funding. 